\title{
The importance of resistance training in the treatment of sarcopenia
}

\author{
Dennis Hunt ${ }^{1}$, Deborah Chapa ${ }^{* 2}$, Barbara Hess ${ }^{1}$, Kathleen Swanick ${ }^{1}$, Andrew Hovanec ${ }^{1}$ \\ ${ }^{1}$ Department of Physical Therapy and Human Performance, Florida Gulf Coast University, Fort Myers, United States \\ ${ }^{2}$ School of Nursing, George Washington University, Washington, United States
}

Received: November 2, 2014

Accepted: December 8, 2014 Online Published: December 21, 2014

DOI: $10.5430 /$ jnep.v5n3p39

URL: http://dx.doi.org/10.5430/jnep.v5n3p39

\begin{abstract}
Utilizing individualized exercise training programs that are inclusive of appropriate resistance training protocols can attenuate the deleterious effects of sarcopenia and enhance quality of life, functional capacity and also diminish its negative effect on independence. Nurses should be educated in how the utilization of proper resistance training (RT) in individuals can be used to ameliorate the effect of sarcopenia, especially considering its relationship to Quality of Life (QoL), functional capacity and independence. In part, this educational process should include how to refer patients to an appropriate healthcare provider trained in assessing sarcopenia and the use of resistance training. However, it is especially important that the provider be an expert on RT that enhances lean muscle, muscular strength, muscular endurance, power, balance and stamina. Therefore, it is vital that nurses have an opportunity to define and recognize how resistance training can be prescribed, ultimately for primary prevention but also as an effective treatment module in enhancing the health outcomes related to QoL, functional capacity and independence.
\end{abstract}

Key Words: Sarcopenia, Resistance training, Functional capacity, Exercise, Independence, Quality of life

\section{Introduction}

Sarcopenia or the involuntary loss of lean muscle mass occurs commonly with aging, beginning as early as age 40 and becoming much more pronounced after age $60 .{ }^{[1]}$ Research suggests that muscle mass can be lost at a rate of $1 \%-2 \%$ per year after the age of 50 years. However, evidence also suggests that strength as a part of muscular fitness declines at a rate greater than the loss of muscle mass. ${ }^{[2]}$ It is postulated that strength declines at a rate of $12 \%-15 \%$ per decade after the 5 th decade of life. ${ }^{[3]}$

Because of the loss of muscle mass and decreases in muscular fitness (i.e. strength, power) which both contribute to quality of life (QoL), functional capacity, and level of independence and reducing the risk of falls in the elderly, it is essential that nurses understand the underlying physiological factors and that resistance training is a possible treatment option for this condition. The purpose of this paper is to describe sarcopenia, as it relates to a patient's health/wellness, reduction of disease risk factors and elucidate an appropriate treatment strategy for the dilemma.

While sarcopenia is not typically considered a disease or pathology it does contribute profoundly to a decline in functional capacity and an increased risk of experiencing a fall in older adulthood. ${ }^{[4]}$ This is especially noteworthy considering that falls are the leading cause of injury death in adults over 65 years of age. ${ }^{[5]}$ Furthermore, research has shown that a decline in functional capacity, associated with sarcopenia, has been shown to negatively affect QoL, perform activities of daily living (ADLs) and maintaining inde-

\footnotetext{
*Correspondence: Deborah Chapa; Email: dchapa@gwu.edu; Address: School of Nursing, George Washington University, 2030 M Street, Suite 300, Washington DC, United States.
} 
pendence in very old age ( $\geq 80$ years of age). ${ }^{[6-8]}$

Some additional detrimental aspects associated with sarcopenia are decreased energy expenditure during rest (i.e. decreased basal metabolic rate) and exercise, leading to the increased potential of body fat and incidence of obesity, increased dyslipidemia, and a decrease in insulin sensitivity. ${ }^{[3]}$ These precarious effects are also known as risk factors that have shown an increase in the incidence of chronic diseases frequently present in today's sedentary adult population. These diseases consist of, but are not limited to coronary artery disease, arthritis, hypertension, and obesity. Research also shows that type 2 diabetes is independently associated with sarcopenia. Evidence also indicates that sarcopenia may contribute to physical disability and increased metabolic disorders in those with diabetes. ${ }^{[9]}$

It has been suggested that in adults younger than age 70, approximately $10 \%-25 \%$ are sarcopenic and in the age group of individuals over the age of 80 years (i.e. the very old) greater than $40 \%$ of the population can be classified as sarcopenic. ${ }^{[2]}$ As early as 2020, it is estimated that individuals over 65 years of age will account for at least $20 \%$ of the entire United States population. ${ }^{[4]}$ Given the potentially grave consequences of sarcopenia, as it relates to loss of functional ability in activities of daily living, lowered quality of life, health implications, independence and risk for experiencing falls, it is now becoming increasingly vital to determine germane and pragmatic interventions that can minimize or reverse the effects of sarcopenia. ${ }^{[6,8]}$

Nurses commonly recognize three types of prevention: primary, secondary, and tertiary. ${ }^{[10]}$ The American Physical Therapy Association (APTA) defines primary prevention as "prevention of a disease in a susceptible population or potentially susceptible population through specific measures such as general health promotion efforts". ${ }^{[10]}$ Other healthcare professionals define primary prevention similarly; for instance, the American Occupational Therapy Association defines primary prevention as "education or health promotion strategies designed to help people avoid the onset of unhealthy conditions, diseases, or injuries".[10] Perhaps most simply stated, primary prevention can be described as an effort to stop a potentially debilitating health issue before it starts. The APTA has defined secondary prevention as "efforts to decrease duration of illness, severity of disease, and sequelae through early diagnosis and prompt intervention". ${ }^{[10]}$ This aspect of prevention relies heavily on the ability of a healthcare practitioner to screen for and diagnose health concerns before they become clinically apparent, as well as the ability to utilize efficacious intervention strategies in a timely manner. The third type of prevention, tertiary prevention, is defined by the APTA in terms of the "efforts to decrease the degree of disability and promote rehabilitation and restoration of function in patients with chronic and irreversible diseases". ${ }^{[10]}$ An example of tertiary intervention is blood glucose control for patients with diabetes.
Each type of prevention is crucial to the provisions of contemporary healthcare. ${ }^{[11]}$

Exercise, including resistance training (i.e. strength and power training) for various intervals of time has been used as an intervention strategy in primary, secondary, and tertiary prevention for many issues including chronic diseases and musculoskeletal health concerns in diverse populations including older adults. ${ }^{[10]}$ Further research in the area of exercise programming needs to be completed in order to identify effective, evidence-based methods that will likely be needed and used to treat the large and growing number of individuals who have been identified as having sarcopenia or who are at risk for sarcopenia and its related disabilities. The level of prevention as well as the health condition or pathology that is being taken care of should determine the resistance training outcome goals (e.g. training for muscular endurance, strength, or power). For instance, muscular endurance training may be indicated as a secondary prevention intervention in a disease process that causes fatigue with activity and an interruption in performing ADLs.

Briefly, in the very old adult ( $\geq 80$ years of age) population a progressive resistance training (PRT) model is not always utilized. This type of model (i.e. PRT) typically emphasizes a progressive overload strategy, which gradually increases the amount of resistance an individual performs an exercise with, while decreasing the number of repetitions performed and has as its overall health outcome the improvement of muscular fitness. ${ }^{[12]}$

\section{Defining sarcopenia}

There are multiple methods described in the literature on how to define and classify sarcopenia. Three of those methods are:

- Appendicular lean mass divided by height squared,

- Residuals method,

- Skeletal mass index (SMI). ${ }^{[13,14]}$

In the past Sarcopenia has been defined by using dual energy x-ray absorptiometry. Initially, x-ray absorptiometry determined appendicular lean mass which was divided by height square to classify an individual as sarcopenic. ${ }^{[13,15]}$ However, Delmonico and colleagues ${ }^{[12]}$ described a method referred to as the residuals method that defined sarcopenia using x-ray absorptiometry to determine both appendicular lean mass, and fat mass, along with using an individual's height. This method differed from previous attempts that only identified sarcopenia by considering the appendicular lean mass divided by height squared. ${ }^{[13,15,16]}$

Individuals were classified as sarcopenic if their appendicular lean mass divided by height squared fell into the sexspecific lowest $20 \%$ of the index distribution. Appendicular lean mass was calculated as the sum of lean mass in the 
arms and legs, assuming that all nonfat and non-bone tissue is skeletal muscle. ${ }^{[13]}$

Delmonico and colleagues ${ }^{[13]}$ were also able to show that individuals characterized as sarcopenic by the residuals method were more likely to show decreased physical performance (i.e., gait speed, chair stands, and standing balance) and self-reported lower performance scores than individuals classified as sarcopenic by only using the appendicular lean mass divided by height squared method. The reason theorized for the performance difference was that the appendicular lean mass divided by height squared method did not take into account body composition (i.e., lean body mass and fat mass) and wrongly categorized many thin individuals as sarcopenic while wrongly identifying overweight individuals as not being sarcopenic. ${ }^{[13]}$ Other research has supported this notion that the relationship of fat and lean mass has vital importance to the incidence of functional limitations and should be taken into account when objectively defining sarcopenia. ${ }^{[17,18]}$ The point of demarcation ${ }^{[13]}$ used to classify sarcopenia utilizing the residuals method for men was less than $7.25 \mathrm{~kg} / \mathrm{ht}^{2}$ and for women less than $5.67 \mathrm{~kg} / \mathrm{ht}^{2}$ (see Table 1).

Table 1: Cut points for classification of sarcopenia

\begin{tabular}{lll}
\hline Study & Men & Women \\
Delmonico $^{[12]}$ & $7.25 \mathrm{~kg} / \mathrm{m}^{2}$ & $5.67 \mathrm{~kg} / \mathrm{m}^{2}$ \\
Fiatarone-Singh $^{[13]}$ & $9.5 \mathrm{~kg} / \mathrm{m}^{2}$ & $7.0 \mathrm{~kg} / \mathrm{m}^{2}$ \\
\hline
\end{tabular}

Other researchers have gone on to utilize bioelectrical impedance assessment (BIA) techniques to extrapolate cutpoints for defining sarcopenia. ${ }^{[14,19]}$ Fiatarone-Singh and colleagues ${ }^{[14]}$ used BIA to calculate what they termed skeletal mass index (SMI). Skeletal Mass Index was calculated by dividing whole-body skeletal muscle mass by height in meters squared. Sarcopenia then was defined as a SMI less than $9.5 \mathrm{~kg} / \mathrm{m}^{2}$ for men and less than $7.0 \mathrm{~kg} / \mathrm{m}^{2}$ for women. This method differs from the Delmonico and colleagues. ${ }^{[13]}$ Study cited previously that only utilized the appendicular lean mass method to classify sarcopenia. The use of whole body skeletal muscle mass in contrast to only upper extremity and lower extremity muscle mass partially explains the difference in the demarcation points for sarcopenia between the two studies.

\section{Physiological factors in sarcopenia}

Sarcopenia is thought to impact many physiological and daily functions. The loss of muscle mass, strength and power associated with sarcopenia has also been linked to increased incidence of falls among older individuals. Additionally, research suggests that the loss of muscular fitness may also be attributed to Type II muscle fiber atrophy. ${ }^{[1,20,21]}$ Type II muscle fibers are largely responsible for muscular endurance. ${ }^{[12]}$ Some atrophy may be the result of inactivity but other issues such as reduced protein turnover, reduced endocrine function, and neuromuscular denervation have been implicated in research findings. ${ }^{[1]}$ Numerous agerelated sensory changes have also been documented regarding the incidence of falls including negative changes in response to tactile stimuli, decreased acuity of joint position, reduced efficiency in motor planning, and decreased sensory nerve conduction velocity in both median and sural nerves. ${ }^{[22]}$ Furthermore, it is important to note that impaired contraction velocity and lower leg power have been shown to be contributors of poorer functional performance. ${ }^{[22]}$

Environmental versus biological aging are contemporary topics among healthcare professionals working with an increasingly older population. Biological aging or chronological aging can refer to genetic, cellular, and molecular factors and processes that affect the course of aging. Environmental aging generally refers to lifestyle factors, including diet, exercise, and activity level that can influence the extent of chronological aging. ${ }^{[23]}$ Arts and colleagues. ${ }^{[20]}$ performed a longitudinal study that included analyzing muscle size (i.e. cross sectional area) over the course of a lifetime that did not provide any lifestyle or exercise intervention. The investigators in the study measured the circumference of both the biceps brachi and quadriceps femoris. Their findings indicated muscle size peaked between the ages of 25 and 50 years of age, but shortly after the age of 50 years, muscle size decreased and the effects of sarcopenia were realized. Their results also demonstrated that the quadriceps muscle size decreased by $50 \%$ in men and $30 \%$ in women between the age of 40 and 90 years. In addition, the Biceps brachi muscle size was shown to decrease by $30 \%$ in men and $20 \%$ in women during that same period. These findings are valuable in gaining a greater perspective of the potentially debilitating process of sarcopenia in the life cycle. ${ }^{[20]}$

\section{Lifestyle, exercise intervention and sar- copenia}

Research suggests that strength-training interventions which address sarcopenia have also been successful in decreasing the risk of falling in an older population. ${ }^{[23]}$ For example, exercises that target ankle and hip strength have been shown to lower the risk of falling as measured by the incidence of falls among participants three months post-intervention as determined by functional independence measure scores, and the Fall Efficacy Scale. ${ }^{[24]}$ Deley and colleagues ${ }^{[25]}$ investigated the effects of a one-year aerobic endurance and resistance training program on 40 healthy adults over 70 years of age. The use of a combined training program which included three hours a week of exercise included 24 participants and a control group of 16 participants who did not receive the training program. The findings of this type of exercise training program study showed that the 24 participants involved in the exercise intervention improved in several measures including strength, aerobic capacity, and field test performances. ${ }^{[25]}$ 
Iglay and colleagues ${ }^{[26]}$ found that individuals between the ages of 61 and 86 years old could experience strength gains between 28\%-34\% from initial strength values after completing a strength program. An interesting variable that was also investigated in their study was the use of a protein enriched diet that was between $112 \%-150 \%$ of the recommended daily protein intake. Iglay and colleagues ${ }^{[27]}$ suggested that the sarcopenic body can also improve body composition parameters in terms of lean muscle mass through exercise training that is supplemented by a diet with a high protein intake.

Hunter and colleagues ${ }^{[3]}$ advocated low intensity, high velocity contractions at $40 \%$ of $1 \mathrm{RM}$ on at least one day per week for older adults participating in resistance training programming to increase muscular power. High velocity contractions were defined in their study as having the participants perform the concentric phase of an exercise as fast as safely possible. The concentric phase is defined as when the muscle shortens during the training movement. ${ }^{[12]}$ An example of a concentric movement is with a biceps curl exercise, when the weight is lifted through the range of motion. Signorile ${ }^{[27]}$ suggests that what other investigators have used "moderately heavy" loads or 30\%-50\% of maximal lifts to establish higher contractile speeds, or a fast rate of completion for each repetition. This faster rate was used to increase the ability of the participants to exert force quickly, in order to improve the muscular fitness component of power. Theoretically, making older individuals more powerful might also improve their functional abilities in dynamic daily activities such as rising from a chair or lifting objects. ${ }^{[27]}$

\section{Implications for nursing practice}

In individuals less then 80 years of age current research has established the efficacy of progressive resistance training as a method to enhance muscle mass and functional capacity. But, in the very old population ( $\geq 80$ years old) additional evidence is needed examining intervention techniques like progressive resistance training as a preventative measure for the risks and issues associated with sarcopenia. A need also exists for this type of research to be completed in order to discern the safest, most efficacious approach to implement a non-pharmacological approach (i.e. exercise intervention strategies) that might attenuate or reverse the effects of sarcopenia on the very old ( $\geq 80$ years old). Sarcopenia may be an element of the "normal" aging process but limiting or reducing its potentially deleterious effects can provide the mounting number of older individuals and the very old with a higher quality life, functional capacity, and independence for a longer period of time. ${ }^{[6,8]}$ Studies previously identified suggest that very old individuals might indeed be able to benefit from resistance training similar to the way the younger population benefits from the same kind of training.

Finally, due to the relationship of sarcopenia to chronic disease, nurses should be comfortable in referring patients to an appropriate healthcare provider trained in prescribing individualized appropriate exercise guidelines for resistance training. Nurses should also be able to educate their patients on the importance of resistance training and its potential positive impact related to their specific condition. When performed correctly, utilizing appropriate resistance training sarcopenia might be attenuated, diminishing its effect on independence, and improving their quality of life and functional capacity. ${ }^{[6,8]}$

\section{Conclusion}

The information in this article illustrates that it is essential for nurses to understand the importance of exercise, specifically resistance training and the need for it to be appropriately prescribed, hopefully as a primary prevention strategy. However, it is vital for nurses to educate their patients especially those with sarcopenia about the potential that performing appropriate individualized resistance training can have on issues related to their condition. If this can be accomplished successfully there is emerging evidence that suggests patient outcomes can be improved. In addition there is robust research that suggests that resistance training can attenuate falls in older individuals, potentially decreasing morbidity and extending life.

\section{Conflicts of Interest Disclosure}

The author declares that there is no conflict of interest statement.

\section{References}

[1] Deschenes MR. Effects of aging on muscle fiber type and size. Sports Med. 2004 Oct; (34); 809-34. PMid:15462613 http://dx .doi.org/10.2165/00007256-200434120-00002

[2] Geithner C McKenney D. Strategies for aging well. J Strength Cond Res. 2010; 32 (5): 36-52. http://dx.doi.org/10.1519/SSC. 0 b013e3181d9a66c

[3] Hunter G, McCarthy J, Bamman, M. ffects of resistance training on older adults. Sports Med. 2004; 34(5): 329-348. http://dx.doi .org/10.2165/00007256-200434050-00005

[4] Silcott L. Sarcopenia: The Silent Crippler. Vib Life. 2008 Sept; 25: $12-14$.

[5] Rose D Fall Proof. Human Kinetics. Champaign, IL. 2003.

[6] Chien M KuoHK WuHT. Sarcopenia, cardiopulmonary fitness, and physical disability in community-dwelling elderly people. Phys. Ther 2010 Sept; 90(9): 1277-87. PMid:20616117 http://dx.doi .org/10.2522/ptj. 20090322 
[7] Katula JA Rejeski WJ MarshAP Enhancing the quality of life in older adults: A comparison of muscular strength and power training. Health Qual Life Outcomes. 2008 June; 6: 45. PMid:18554394 http://dx.doi.org/10.1186/1477-7525-6-45

[8] Liu CJ Latham NK Progressive resistance strength training for improving physical function in older adults. Cochrane Database Syst Rev. 2009; (3).

[9] Kim T Park M Choi K et al. Prevalence and determinant factors of sarcopenia in patients with type 2 diabetes; The Korean Sarcopenic Obesity Study (KSOS). Diabetes Care. 2010 Apr; 33(7):1497-1499. PMid:20413515 http://dx.doi .org/10.2337/dc09-2310

[10] Fair SE Wellness and Physical Therapy. Jones \& Bartlett Publishers. Sudbury, MA. 2011.

[11] HHS: Strategic goal one: strengthen healthcare. Washington, DC. Health and Human Services cMarch 102014 [cited 2014 December 6]. Available from: http://www.hhs.gov/strategic-plan/go al1.html

[12] Baechle T Earle R. Essentials of Strength Training and Conditioning. Human Kinetics. Champaign,IL. 2000. PMid:11133817

[13] Delmonico M Harris T Lee J et al. Alternative definitions of sarcopenia, lower extremity performance, and functional impairment with aging in older men and women. J Am Geriats Soc. 2007 June; 55: 769-74. PMid:17493199 http://dx.doi.org/10.1111/j.1 532-5415.2007.01140.x

[14] Fiatarone Singh M Singh N HansenR et al. Methodology and baseline characteristics for the Sarcopenia and Hip Fracture study: a 5year prospective study. J Gerentol A Biol Sci Med Sci. 2009 Feb; 64A(5): 568-574.

[15] Bunout D de la Maza M Barrera G et al. Assessment of sarcopenia: longitudinal versus cross-sectional body composition data $\mathrm{Ag}$ Clin Experi Res. 2007 Aug; 19 (4): 295-99.

[16] Estrada M Kleppinger A Judge J et al. Functional impact of relative versus absolute sarcopenia in healthy older women. J Am Geriatr Soc. 2007 Nov; 55: 1712-19. PMid:17979895 http://dx.doi.o $\mathrm{rg} / 10.1111 / \mathrm{j} .1532-5415.2007 .01436 . \mathrm{x}$

[17] Jensen GL Influence of sarcopenia on the development of physical disability: The cardiovascular health study. J Am Geriatr Soc. 2005
Jan; 54: 56-62. PMid:16420198 http://dx.doi.org/10.1111 /j.1532-5415.2005.00540.x

[18] Visser M Langlois J Guralnik J High body fatness, but not low fatfree mass, predicts disability in older men and women. The Cardiovascular Health Study. Am J Clin Nutr. 1998 Sept; 68: 584-590. PMid:9734734

[19] Castaneda C Janssen I.Ethnic comparisons of sarcopenia and obesity in diabetes. Ethn Dis. 2005 Aut; 15 : 664-670.

[20] Arts I Pillen S Overeem,S et al. Rise and fall of skeletal muscle size over the entire life span. J Am Geriatr Soc. 2007 Jul; 55(7): 1150-2. PMid:17608902 http://dx.doi.org/10.1111/j.1532-5415. $2007.01228 . x$

[21] Lexell, J.Strength training and muscle hypertrophy in older men and women. Top Geriatri Rehabil. 2000 Mar; 15(3): 41-46. http: //dx.doi.org/10.1097/00013614-200003000-00007

[22] O'Sullivan S B Schmitz T.J. Physical Rehabilitation 5th ed. Philadelphia, PA. F.A. Davis. 2007.

[23] Hurley B Roth S Strength training in the elderly: effects on risk factors for age-related diseases. Sports Med. 2000 Oct; 30(4): 249-68. PMid:11048773 http://dx.doi.org/10.2165/00007256-200 030040-00002

[24] Kato M Izumi K. Hiramatsu T Shogenji M. Development of an exercise program for fall prevention for elderly persons in a long term care facility. Jpn J Nurs Sci. 2006 Jul; 3: 107-17. http: //dx.doi.org/10.1111/j.1742-7924.2006.00057.x

[25] Deley G Kervio G Van Hoecke J et al. Effects of a one-year exercise training program in adults over 70 years old: a study with a control group. Aging Clin Exp Res. 2007 Aug; 19(4): 310-15. PMid:17726362 http://dx.doi.org/10.1007/BF03324707

[26] Iglay HB Thyfault JP Apolzan JW Campbell WW. Resistance training and dietary protein: effects on glucose tolerance and contents of skeletal muscle insulin signaling proteins in older persons. Am J Clin Nutr. 2007 Apr; 85: 1005-113. PMid:17413099

[27] Signorile J Developing power in older adults supports independence, prevents falls, and promotes quality of life. J Act Aging. 2005 JanFeb; 35-43. 